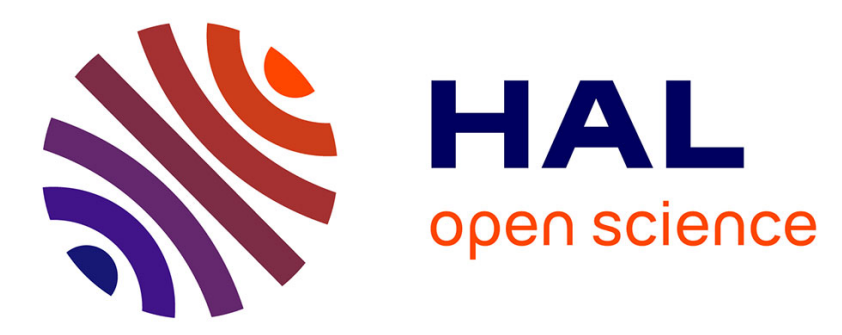

\title{
Resonant Edge Magnetoplasmons and Their Decay in Graphene
}

\author{
N. Kumada, P. Roulleau, B. Roche, M. Hashisaka, H. Hibino, I. Petković, \\ D. c. Glattli
}

\section{> To cite this version:}

N. Kumada, P. Roulleau, B. Roche, M. Hashisaka, H. Hibino, et al.. Resonant Edge Magnetoplasmons and Their Decay in Graphene. Physical Review Letters, 2014, 113 (26), pp.266601. 10.1103/PhysRevLett.113.266601 . cea-01409225

\section{HAL Id: cea-01409225 https://hal-cea.archives-ouvertes.fr/cea-01409225}

Submitted on 5 Dec 2016

HAL is a multi-disciplinary open access archive for the deposit and dissemination of scientific research documents, whether they are published or not. The documents may come from teaching and research institutions in France or abroad, or from public or private research centers.
L'archive ouverte pluridisciplinaire HAL, est destinée au dépôt et à la diffusion de documents scientifiques de niveau recherche, publiés ou non, émanant des établissements d'enseignement et de recherche français ou étrangers, des laboratoires publics ou privés. 


\title{
Resonant Edge Magnetoplasmons and Their Decay in Graphene
}

\author{
N. Kumada, ${ }^{1,2,{ }^{*}}$ P. Roulleau, ${ }^{2}$ B. Roche,${ }^{2}$ M. Hashisaka,${ }^{3}$ H. Hibino, ${ }^{1}$ I. Petković, ${ }^{2}$ and D. C. Glattli ${ }^{2}$ \\ ${ }^{1}$ NTT Basic Research Laboratories, NTT Corporation, 3-1 Morinosato-Wakamiya, Atsugi 243-0198, Japan \\ ${ }^{2}$ Nanoelectronics Group, Service de Physique de l'Etat Condensé, IRAMIS/DSM (CNRS URA 2464), CEA Saclay, \\ F-91191 Gif-sur-Yvette, France \\ ${ }^{3}$ Department of Physics, Tokyo Institute of Technology, Ookayama, Meguro, Tokyo 152-8551, Japan
}

(Received 2 July 2014; published 22 December 2014)

\begin{abstract}
We investigate resonant edge magnetoplasmons (EMPs) and their decay in graphene by high-frequency electronic measurements. From EMP resonances in disk shaped graphene, we show that the dispersion relation of EMPs is nonlinear due to interactions, giving rise to the intrinsic decay of EMP wave packets. We also identify extrinsic dissipation mechanisms due to interaction with localized states in bulk graphene from the decay time of EMP wave packets. We indicate that, owing to the linear band structure and the sharp edge potential, EMP dissipation in graphene can be lower than that in GaAs systems.
\end{abstract}

Edge channels (ECs) in quantum Hall $(\mathrm{QH})$ states provide unique chiral one-dimensional systems to perform a variety of electron quantum experiments including electronic Mach-Zehnder interferometry [1,2] and flying qubits $[3,4]$. ECs support collective excitations called edge magnetoplasmons (EMPs) [5,6], which form the bosonic modes in a Tomonaga-Luttinger liquid representation [7]. EMPs lead to charge fractionalization $[8,9]$ and their dissipation causes energy relaxation $[10,11]$ and decoherence $[4,12,13]$ in ECs. To better understand these physics and to obtain robust quantum effects, investigation of EMP decay mechanism is essential. EMPs in graphene are of particular interest because, owing to the linear and gapless band structure of graphene, their dissipation would be lower than other conventional two-dimensional systems. However, although EMPs in graphene have been investigated [14-20], fundamental quantities such as dispersion and decay are yet to be measured. Furthermore, to the best of our knowledge, the decay mechanism of EMPs in any two-dimensional systems has never been identified.

In this Letter, we identify the dispersion relation and dissipation mechanisms of EMPs using electronic techniques with the frequency range up to $65 \mathrm{GHz}$. We prepared disk shaped graphene devices and measured high-frequency transmission in frequency and time domain. Frequency domain measurement gives resonant frequencies of EMPs, from which the dispersion relation is obtained. EMP decay time $\tau$ is directly measured in time domain. From the frequency and temperature dependence of $\tau$, we identify that dissipation is caused by interaction with localized states in bulk graphene. The quality factor of our EMP devices is larger than a reported value in GaAs systems [21]. We suggest that the lower decay is an intrinsic property of graphene, stimulating quantum transport experiments, and plasmonic applications [22] using graphene.
We used graphene grown by thermal decomposition of a $6 H$-SiC (0001) substrate (Fig. 1). Graphene is etched into a disk shape. We used two devices with the perimeter $P$ of 200 and $1000 \mu \mathrm{m}$ called 200- and 1000- $\mu \mathrm{m}$ samples, respectively. The carrier is electron and the density is about $5 \times 10^{11} \mathrm{~cm}^{-2}$. The mobility is about $12000 \mathrm{~cm}^{2} / \mathrm{V} \mathrm{s}$. The surface of graphene was covered with 100-nm-thick hydrogen silsesquioxane (HSQ) and 60-nm-thick $\mathrm{SiO}_{2}$ insulating layers. Two high-frequency lines to inject and detect EMPs were deposited on top of the insulating layer, separating the perimeter in the ratio of $3: 1$. The overlap between the injector (detector) and graphene are $3 \mu \mathrm{m}$ in width. In a $\mathrm{QH}$ effect regime at high perpendicular field $B$, the active length of the injector and the detector corresponds to the width of $\mathrm{ECs}$, which is roughly estimated to be $10 \mathrm{~nm}$ in a steep edge potential. Then the impedance of the injector and the

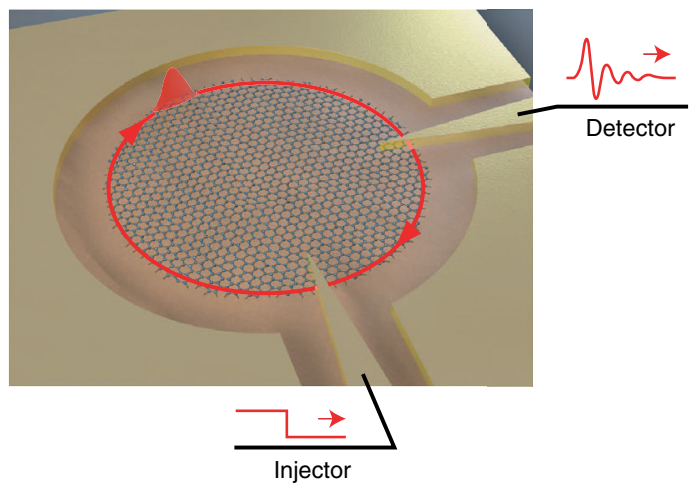

FIG. 1 (color online). Schematic of the device. Disk shaped graphene is covered with $160 \mathrm{~nm}$ thick insulating layer. The capacitive injector and detector were deposited on top of the insulating layer. The overlap between the injector (detector) and graphene are $3 \mu \mathrm{m}$ in width. A sinusoidal wave or a voltage step is sent to the injector and the current response is detected through the detector. 

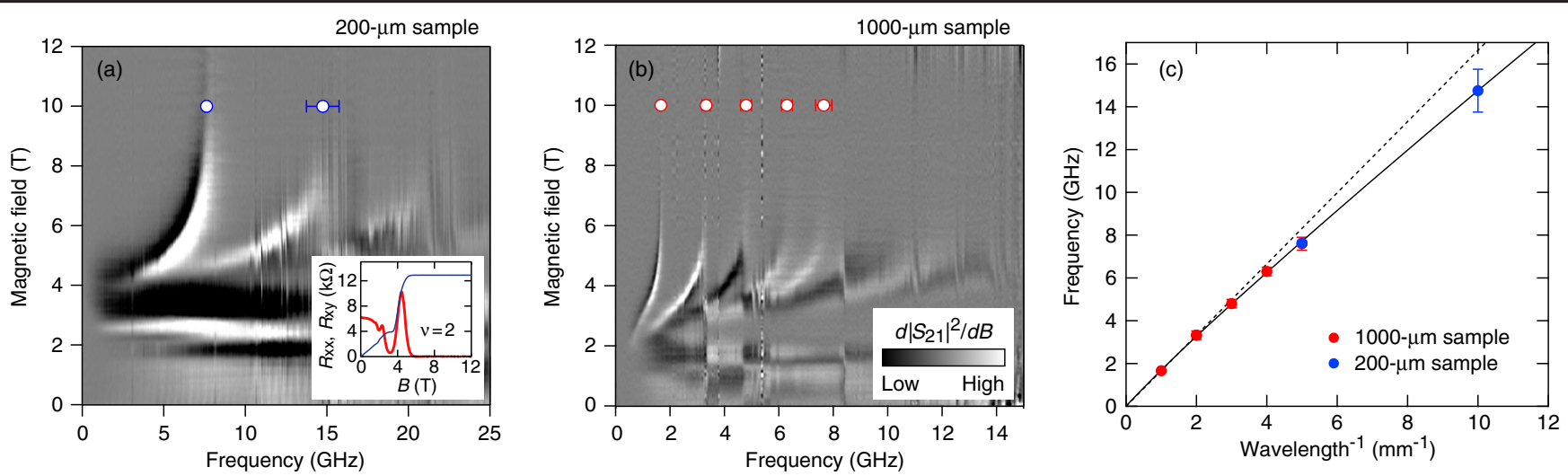

FIG. 2 (color online). Results of frequency domain measurement. (a) and (b) Transmission signals of the 200- and 1000- $\mu$ m samples, respectively. The differentiated transmission power $\left(d\left|S_{21}\right|^{2} / d B\right)$ is plotted as a function of the frequency and $B$. Open dots with error bar indicate the resonant frequency at high $B$ (details for the accurate determination of the resonant frequency are given in [23]). Inset in (a) shows the longitudinal resistance $\left(R_{x x}\right.$; red thick trace) and the Hall resistance ( $R_{x y}$; blue trace) of a Hall bar device fabricated from the same graphene wafer obtained by standard dc measurement. (c) Dispersion relation of the EMP mode: the resonant frequencies [open dots in (a) and (b)] are plotted as a function of $\lambda^{-1}$ determined by Eq. (1). The data point for the 5th harmonics in the $1000-\mu \mathrm{m}$ sample coincides with that for the fundamental mode in the $200-\mu \mathrm{m}$ sample at $\lambda^{-1}=5 \mathrm{~mm}^{-1}$. The solid curve is the dispersion relation given by Eq. (2). The dashed line is the result of the linear fit for small $\lambda^{-1}$ regime, which gives the group velocity $v_{g}=d f / d \lambda^{-1}=1.7 \times 10^{6} \mathrm{~m} / \mathrm{s}$.

detector at $10 \mathrm{GHz}$ is calculated to be $\simeq 3 \mathrm{M} \Omega$ with the dielectric constant and the thickness of HSQ $\left(\epsilon_{\mathrm{HSQ}}=2.8\right.$ and $\left.d_{\mathrm{HSQ}}=100 \mathrm{~nm}\right)$ and those of $\mathrm{SiO}_{2}\left(\epsilon_{\mathrm{SiO}_{2}}=3.9\right.$ and $d_{\mathrm{SiO}_{2}}=60 \mathrm{~nm}$ ). This is much larger than the impedance of the EC, which corresponds to $R_{x y} \sim 10 \mathrm{k} \Omega$ in a QH state, hardly contributing to the EMP dissipation. Magnetic fields up to 12 Tesla have been used. The base temperature is $T=4.2 \mathrm{~K}$.

We first present the frequency domain results. In this measurement, $\left|S_{21}\right|^{2}=P_{\text {out }} / P_{\text {in }}$ is recorded while sweeping the frequency of the sinusoidal voltage applied to the injector at each value of $B$, where $P_{\text {in }}$ and $P_{\text {out }}$ are the injected and detected power, respectively. Figures 2(a) and 2(b) plot the differential of the signal with respect to $B\left(d\left|S_{21}\right|^{2} / d B\right)$; by differentiating, we reject $B$ independent cross talk between the high-frequency lines and highlight the signal associated with EMPs. For $B \gtrsim 6 \mathrm{~T}$, EMP resonances, manifested as a dip and peak structure in $d\left|S_{21}\right|^{2} / d B$ appear with almost equal frequency spacing. The spacing is about 7.6 and $1.6 \mathrm{GHz}$ for the 200- and $1000-\mu \mathrm{m}$ samples, respectively, roughly inversely proportional to the perimeter. As $B$ is decreased, the resonances shift to lower frequency and then are quickly damped.

The EMP resonances can be understood as follows. The $B$ range for the sharp resonances corresponds to the Landau level $\nu=2 \mathrm{QH}$ state, in which the longitudinal resistance $R_{x x}$ is vanishing [inset of Fig. 2(a)]. In this condition, scattering of EMPs by charges in the bulk graphene is suppressed and EMPs orbit along the graphene edge. Then the resonance occurs when the wavelength of the EMP mode corresponds to [25],

$$
\lambda=\frac{P}{j},
$$

where $j$ is an integer. EMPs with $\lambda(j=1)$ is the fundamental mode and $\lambda(j \geq 2)$ is the $j$ th harmonics. From the resonant frequency $f$, the phase velocity is obtained as $v_{p}=f P / j$. The decrease in $f$ and the broadening of the resonance feature on the lower $B$ side of the $\nu=2 \mathrm{QH}$ state are due to the decrease in $v_{p}$ induced by interaction between EMPs and charges in bulk graphene (details are discussed in Supplemental Material [23]). For further smaller $B, \mathrm{QH}$ effect is not well developed and injected charges diffuse rather than propagate as EMPs $[5,26]$. The broad signal around $B=3 \mathrm{~T}$ suggest that the diffusion and EMP propagation coexist in the $\nu=6 \mathrm{QH}$ state with finite $R_{x x}$.

Here, we focus on the resonant modes in the $\nu=2 \mathrm{QH}$ state at high $B$. In Fig. 2(c), the frequency of the fundamental and harmonic modes are plotted as a function of $\lambda^{-1}$ determined by Eq. (1); the plot represents the dispersion relation. The dispersion is almost linear for smaller $\lambda^{-1}$ with the group velocity $v_{q}=d f / d \lambda^{-1}=1.7 \times$ $10^{6} \mathrm{~m} / \mathrm{s}$ [dashed line in Fig. 2(c)] [18,19]. As $\lambda^{-1}$ is increased, the dispersion deviates from the linear line and $v_{g}$ decreases gradually. This behavior can be reproduced by theory [5] for EMPs in a hard-wall edge potential,

$$
f=\left[\frac{\sigma_{x y}}{2 \pi \epsilon^{*} \epsilon_{0}}\left(\ln \frac{2}{2 \pi \lambda^{-1} w}+1\right)+v_{D}\right] \lambda^{-1},
$$

where $\sigma_{x y}$ is the Hall conductance, $\epsilon^{*}$ is the effective dielectric constant, $v_{D}$ is the drift velocity, and $w$ is the transverse width of EMPs. The logarithmic term coming from interactions 
is responsible for the nonlinear dispersion. The best fit [solid curve in Fig. 2(c)] gives $v_{D}=(5 \pm 1) \times 10^{5} \mathrm{~m} / \mathrm{s}$ and $\epsilon^{*}=14.3 \pm 1.4$. The value of $v_{D}$ scales with the Fermi velocity $v_{F}=10^{6} \mathrm{~m} / \mathrm{s}$, consistent with previous experiments [19]. $w$ is calculated to be $4 \mathrm{~nm}$ [23], which is much smaller than $\sim 2 \mu \mathrm{m}$ in GaAs/AlGaAs heterostructures, where $w$ is determined by the shape of the slowly varying edge potential $[27,28]$.

Next, we show time domain results, where a EMP wave packet is generated by a voltage step applied to the injector and then the signal $S$ at the detector is recorded as a function of time [21]. The rise time of the voltage step is $\Delta_{t}=11 \mathrm{ps}$. Slight increase in $\Delta_{t}$ at the injector being taken into account, the spatial width of the EMP wave packet is estimated to be $\Delta_{s}=\Delta_{t} \times v_{p} \sim 40 \mu \mathrm{m}$. Figure 3(a) shows the time trace of the differential signal $d S / d B$ for the $200-\mu \mathrm{m}$ sample. For $B \gtrsim 6 \mathrm{~T}$, the data clearly show the periodic oscillations with decay. The phase of the signal reflects the chirality of the EMP motion. Since the injector and the detector separate the perimeter in the ratio of $3: 1$, a $\pi$ phase shift occurs when the chirality is changed by changing the direction of $B$ [inset of Fig. 3(a)]. This $\pi$ phase shift allows us to eliminate the cross talk without the differentiation: by subtracting the signal for opposite direction but the same amplitude of $B$, the cross talk is eliminated while the EMP signal is increased twofold. Figure 3(b) shows the result of the subtraction. From this plot, the frequency and the decay time of the EMP orbital motion can be directly obtained.

Figure $4(\mathrm{a})$ is the cross section at $|B|=10 \mathrm{~T}$, from which $f=7.64 \mathrm{GHz}$ and the EMP decay time $\tau=353 \mathrm{ps}$ are obtained. The value of $f$ corresponds to that of the fundamental mode [Fig. 2(a)]. Similar measurement for the $1000-\mu \mathrm{m}$ sample shows $f=1.67 \mathrm{GHz}$ and $\tau=2.93 \mathrm{~ns}$ [Fig. 4(b)]. These results show that $\tau$ grows with decreasing $f$. The $f$ dependence of $\tau$ changes qualitatively for higher $T$. At $T=30 \mathrm{~K}, \tau$ becomes smaller $\sim 200 \mathrm{ps}$ for both samples [insets of Figs. 4(a) and 4(b)]. Detailed $T$ dependence of $\tau$ in the 200- $\mu \mathrm{m}$ sample shows that, although $\tau$ increases with decreasing $T$, it is limited at around $400 \mathrm{ps}$ [Fig. 4(c)]. These results indicate that the primary mechanism of the EMP dissipation changes with $T$ : the $f$ dependent term determines the dissipation at low $T$, while the $f$ independent contribution increases with $T$.

The behavior of $\tau$ can be explained by a model based on the coupling to localized states [Fig. 4(e)], which are conductive channels along contours of disorder potential [29]. The localized states couple to EMPs capacitively and resistively. In a circuit representation, the EC is modeled as a unidirectional transmission line with impedance $Z=R_{x y}$ and channel capacitance $C_{\mathrm{ch}}$ [30]. The resistive coupling to the localized states corresponds to a shunt resistor with a conductance $\sigma_{x x} \sim R_{x x} / R_{x y}^{2}$. The dissipation by the shunt resistor $\tau^{-1} \propto \sigma_{x x} / C_{\mathrm{ch}}$ is almost independent of $f$, while it increases with $T$. On the other hand, the capacitive coupling
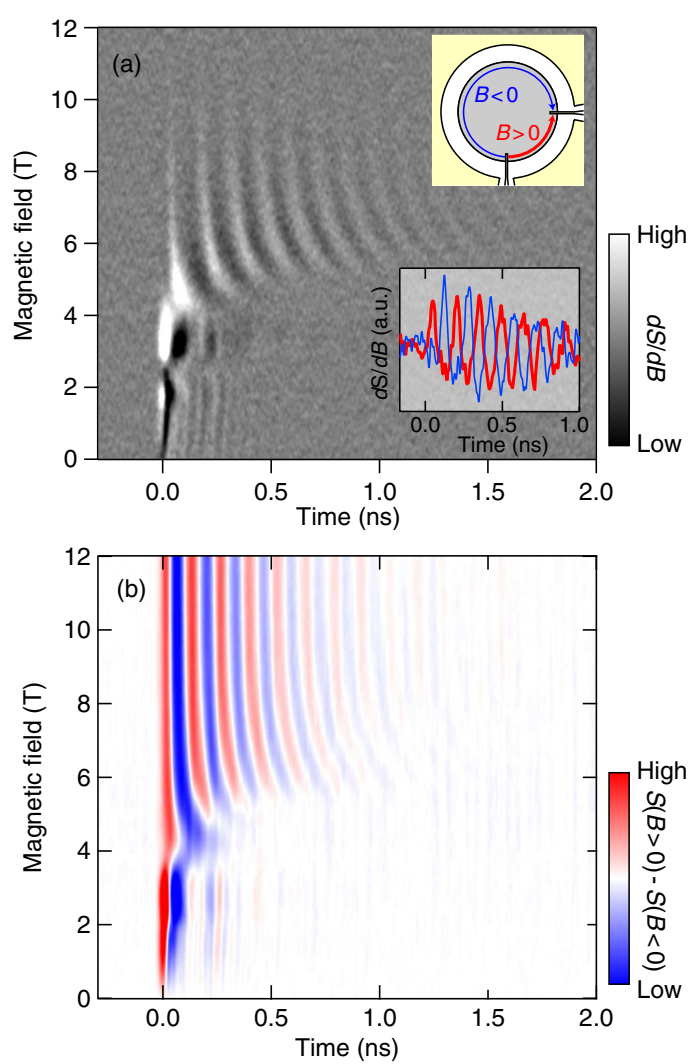

FIG. 3 (color online). Results of time domain measurement. (a) Differential signal $(d S / d B)$ of the $200-\mu \mathrm{m}$ sample a function of time and $B$. Inset shows $d S / d B$ at $B=6.5 \mathrm{~T}$ (red thick trace) and $B=-6.5 \mathrm{~T}$ (blue trace). The chirality of the EMP orbital motion is counterclockwise (clockwise) for positive (negative) $B$. (b) $S(B>0)-S(B<0)$ for the $200-\mu \mathrm{m}$ sample.

can be represented by a shunt capacitor $C_{\mathrm{loc}}$. The effect of charge excitations within the localized states can be included as a series resistor $R_{\mathrm{loc}}$. The dissipation through the capacitive coupling increases with the admittance of the capacitor $2 \pi f C_{\text {loc }}$; more precisely, the dissipation is $\tau^{-1} \propto R_{\mathrm{loc}}\left(C_{\mathrm{loc}} f\right)^{2} /\left(C_{\mathrm{ch}}+C_{\mathrm{loc}}\right)$ [23]. Since $C_{\mathrm{loc}}$ is determined by the geometry independent of $T$, this term becomes important at low $T$. To confirm our model, we simulated the time evolution of the EMP wave packet [23] assuming $\tau^{-1}=\alpha f^{2}+\beta(T)$. In the simulation, the dispersion relation obtained by frequency domain measurements is included. The simulation with $\beta=0$ reproduces the experimental results at $T=4 \mathrm{~K}$ [black traces in Figs. 4(a) and 4(b)], indicating that the capacitive coupling is the main source of the decay at low $T$. The $\alpha$ values used are $4.9 \times 10^{-12}$ and $1.2 \times 10^{-11}$ for the 200 - and $1000-\mu \mathrm{m}$ samples, respectively. The difference of $\alpha$ values would be due to sample dependence of $C_{\mathrm{loc}}$ and $R_{\mathrm{loc}}$. Data for higher $T$ can also be reproduced with temperature dependent $\beta$ and the constant $\alpha$ [black traces in the insets of Figs. 4(a) and 4(b)]. The $T$ dependence of $\beta$ follows the variable range hopping law [31], 

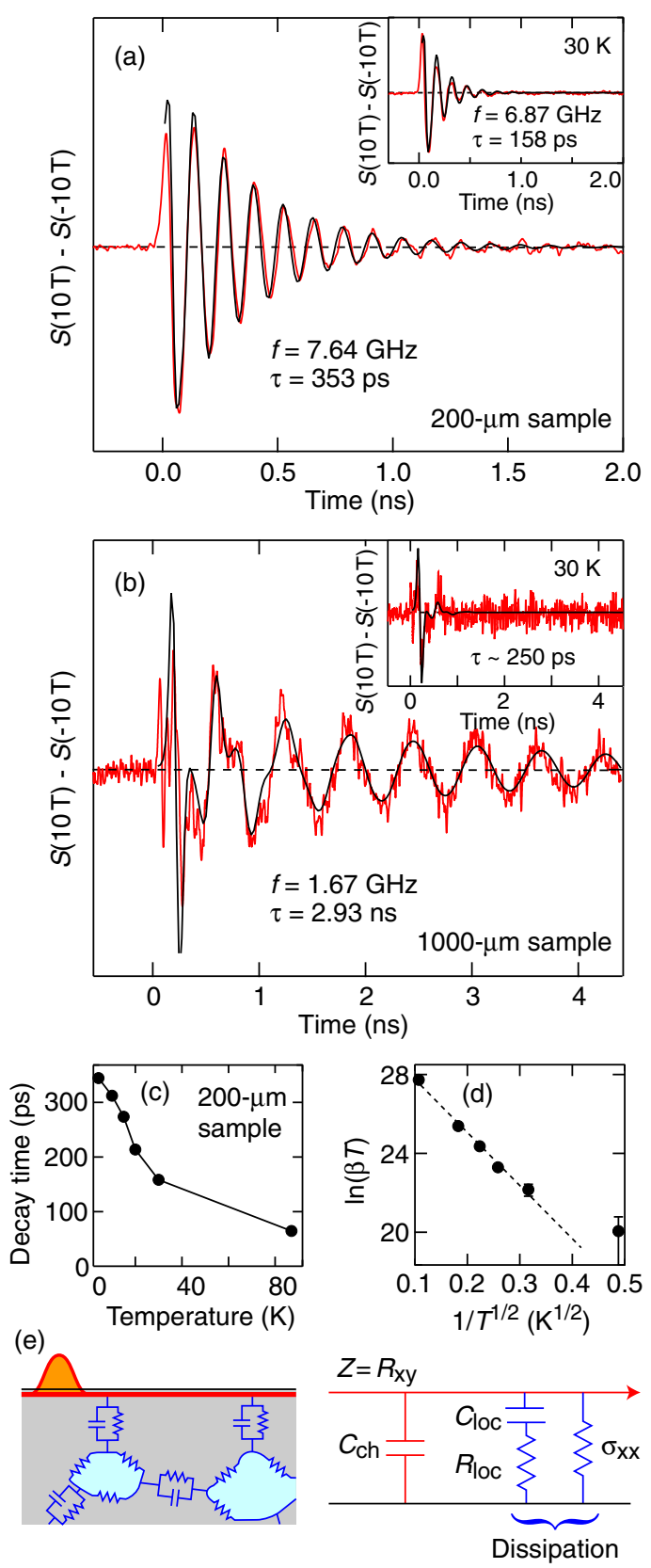

FIG. 4 (color online). Temperature dependence of EMP decay. (a) and (b) $S(10 \mathrm{~T})-S(-10 \mathrm{~T})$ at $T=4 \mathrm{~K}$ for the 200 - and $1000-\mu \mathrm{m}$ samples, respectively. Insets show the traces at $T=30 \mathrm{~K}$. Black traces represent results of the simulation (details are indicated in the main text). (c) $\tau$ for the $200-\mu \mathrm{m}$ sample as a function of $T$. (d) $\ln (\beta T)$ as a function of $T^{-1 / 2}$. Linear fit gives $T_{0}^{1 / 2}=26.9 \pm 1.4 \mathrm{~K}^{1 / 2}$ in Eq. (3). (e) Illustration and equivalent circuit model for the EMP dissipation through interaction with localized states in the interior of graphene.

$$
\beta \propto \frac{1}{T} \exp \left[-\left(T_{0} / T\right)^{1 / 2}\right]
$$

with a characteristic temperature $T_{0} \sim 700 \mathrm{~K}$ for the hopping between localized states [Fig. 4(d)]. This value is consistent with that obtained by the temperature dependence of $\sigma_{x x}$
[32], supporting that the $T$ dependent term comes from the resistive coupling. It is worth noting that the simulation also reproduces fine peaks and dips that appear in the 1000- $\mu \mathrm{m}$ sample for smaller time $<1 \mathrm{~ns}$. The fine structures in the simulation comes from orbital motions of the EMP pulse with the initial width $\sim 40 \mu \mathrm{m}$ much narrower than $P=$ $1000 \mu \mathrm{m}$ [23]. The pulse width becomes broader during the orbital motion by the dispersion until the resonance mode is formed. The agreement between the experiment and the simulation demonstrates that the dispersion relation can also be observed in time domain.

We obtained the quality factor of the resonator $Q=8.5$ and 15.4 at $T=4 \mathrm{~K}$ using $Q=\pi f \tau$ with measured $f$ and $\tau$ for the $200-\mu \mathrm{m}$ and $1000-\mu \mathrm{m}$ samples, respectively. Note that consistent values can be obtained from the peak width of the fundamental mode in frequency domain [23]. These values are larger than $Q \sim 3.8$ obtained by similar time domain measurements using two-dimensional electron systems in GaAs/AlGaAs heterostructures with much higher mobility of $6.2 \times 10^{6} \mathrm{~cm}^{2} / \mathrm{V} \mathrm{s}$ at $f \sim 300 \mathrm{MHz}$ and $T=0.3 \mathrm{~K}$ [21]. We suggest that the smaller decay is an intrinsic property of graphene. Larger cyclotron gap arising from lighter effective mass suppresses the resistive coupling to the localized states. At the same time, it reduces the size of the localized states, reducing the capacitive coupling. Atomically sharp edge potential would also contribute to the smaller decay: narrower $w$ at the sharp edge potential prevents EMPs from being excited inside ECs to acoustic charge modes [33]. Our results indicate that graphene ECs provides a platform for robust quantum effects, stimulating the use of graphene for quantum transport experiments and plasmonic applications.

The ERC Advanced Grant No. 228273 MeQuaNo and the ANR MetroGraph grant are acknowledged. The authors are grateful to F.I. B. Williams, S. Tanabe, and for experimental support of P. Jacques and M. Ueki.

*kumada.norio@lab.ntt.co.jp

[1] Y. Ji, Y. Chung, D. Sprinzak, M. Heiblum, D. Mahalu, and H. Shtrikman, Nature (London) 422, 415 (2003).

[2] P. Roulleau, F. Portier, D. C. Glattli, P. Roche, A. Cavanna, G. Faini, U. Gennser, and D. Mailly, Phys. Rev. Lett. 100, 126802 (2008).

[3] I. Neder, N. Ofek, Y. Chung, M. Heiblum, D. Mahalu, and V. Umansky, Nature (London) 448, 333 (2007).

[4] E. Bocquillon, V. Freulon, J. M. Berroir, P. Degiovanni, B. Plaçais, A. Cavanna, Y. Jin, and G. Féve, Science 339, 1054 (2013).

[5] V. A. Volkov and S. A. Mikhailov, Zh. Eksp. Teor. Fiz. 94, 217 (1988) [Sov. Phys. JETP 67, 1639 (1988)].

[6] E. Y. Andrei, D. C. Glattli, F. I. B. Williams, and M. Heiblum, Surf. Sci. 196, 501 (1988).

[7] X. G. Wen, Phys. Rev. B 41, 12838 (1990). 
[8] E. Bocquillon, V. Freulon, J. M. Berroir, P. Degiovanni, B. Plaçais, A. Cavanna, Y. Jin, and G. Féve, Nat. Commun. 4, 1839 (2013).

[9] H. Kamata, N. Kumada, M. Hashisaka, K. Muraki, and T. Fujisawa, Nat. Nanotechnol. 9, 177 (2014).

[10] P. Degiovanni, C. Grenier, G. Féve, C. Altimiras, H. le Sueur, and F. Pierre, Phys. Rev. B 81, 121302 (2010).

[11] I. P. Levkivskyi and E. V. Sukhorukov, Phys. Rev. B 85, 075309 (2012).

[12] I. P. Levkivskyi and E. V. Sukhorukov, Phys. Rev. B 78, 045322 (2008).

[13] C. Wahl, J. Rech, T. Jonckheere, and T. Martin, Phys. Rev. Lett. 112, 046802 (2014).

[14] O. G. Balev, P. Vasilopoulos, and H. O. Frota, Phys. Rev. B 84, 245406 (2011).

[15] W. Wang, S. P. Apell, and J. M. Kinaret, Phys. Rev. B 86, 125450 (2012).

[16] J. Crassee, M. Orlita, M. Potemski, A. L. Walter, M. Ostler, T. Seyller, I. Gaponenko, J. Chen, and A. B. Kuzmenko, Nano Lett. 12, 2470 (2012).

[17] H. Yan, Z. Li, X. Li, W. Zhu, P. Avouris, and F. Xia, Nano Lett. 12, 3766 (2012).

[18] N. Kumada, S. Tanabe, H. Hibino, H. Kamata, M. Hashisaka, K. Muraki, and T. Fujisawa, Nat. Commun. 4, 1363 (2013).

[19] I. Petković, F. I. B. Williams, K. Bennaceur, F. Portier, P. Roche, and D. C. Glattli, Phys. Rev. Lett. 110, 016801 (2013).

[20] A. Politano and G. Chiarello, Nanoscale 6, 10927 (2014).
[21] R. C. Ashoori, H. L. Stormer, L. N. Pfeiffer, K. W. Baldwin, and K. West, Phys. Rev. B 45, 3894 (1992).

[22] G. Viola and D. P. DiVincenzo, Phys. Rev. X 4, 021019 (2014).

[23] See Supplemental Material, which includes Ref. [24], at http://link.aps.org/supplemental/10.1103/PhysRevLett.113 .266601 for details about the determination of the resonant frequency, theoretical model, simulations, magnetic field, and temperature dependence data.

[24] A. Bostwick, F. Speck, T. Seyller, K. Horn, M. Polini, R. Asgari, A. H. MacDonald, and E. Rotenberg, Science 328, 999 (2010).

[25] N. Q. Balaban, U. Meirav, H. Shtrikman, and V. Umansky, Phys. Rev. B 55, R13397 (1997).

[26] M. D. Johnson and G. Vignale, Phys. Rev. B 67, 205332 (2003).

[27] N. B. Zhitenev, R. J. Haug, K. v. Klitzing, and K. Eberl, Phys. Rev. B 49, 7809 (1994).

[28] N. Kumada, H. Kamata, and T. Fujisawa, Phys. Rev. B 84, 045314 (2011).

[29] B. Huckenstein, Rev. Mod. Phys. 67, 357 (1995).

[30] M. Hashisaka, H. Kamata, N. Kumada, K. Washio, R. Murata, K. Muraki, and T. Fujisawa, Phys. Rev. B 88, 235409 (2013).

[31] A. L. Efros and B. I. Shklovskii, J. Phys. C 8, L49 (1975).

[32] K. Bennaceur, P. Jacques, F. Portier, P. Roche, and D. C. Glattli, Phys. Rev. B 86, 085433 (2012).

[33] I. L. Aleiner and L. I. Glazman, Phys. Rev. Lett. 72, 2935 (1994). 